\title{
Quantitative Understanding of the Optical Properties of a Single, Complex-Shaped Gold Nanoparticle from Experiment and Theory
}

\author{
Eduardo M. Perassi, ${ }^{\dagger}$ Calin Hrelescu, ${ }^{\ddagger, \perp}$ Andreas Wisnet, ${ }^{\S}$ Markus Döblinger, ${ }^{\S}$ Christina Scheu, ${ }^{\S}$ \\ Frank Jäckel, ${ }^{\neq, l, *}$ Eduardo A. Coronado, ${ }^{\dagger, *}$ and Jochen Feldmann ${ }^{\ddagger, *}$ \\ ${ }^{\dagger}$ Instituto de Investigaciones en Fisico-química de Córdoba (INFIQC), CONICET and Departamento de Fisicoquímica, Fac. de Ciencias Químicas, Universidad Nacional \\ de Córdoba, Córdoba 5000, Argentina, ${ }^{\ddagger}$ Photonics and Optoelectronics Group, Department of Physics and Center for NanoScience (CeNS), Ludwig-Maximilians- \\ Universität München, Amalienstr. 54, 80799 Munich, Germany, ${ }^{5}$ Department of Chemistry and CeNS, Ludwig-Maximilians-Universität München, Butenandtstr. 5-11 (E), \\ 81377 Munich, Germany, ${ }^{\perp}$ Institute of Applied Physics, Johannes Kepler University, 4040 Linz, Austria, and "Department of Physics and Stephenson Institute for \\ Renewable Energy, University of Liverpool, Chadwick Building, Peach Street, Liverpool L69 7ZF, United Kingdom
}

\begin{abstract}
We report on a combined study of Rayleigh and Raman scattering spectroscopy, 3D electron tomography, and discrete dipole approximation (DDA) calculations of a single, complex-shaped gold nanoparticle (NP). Using the exact reconstructed 3D morphology of the NP as input for the DDA calculations, the experimental results can be reproduced with unprecedented precision and detail. We find that not only the exact NP morphology but also the surroundings including

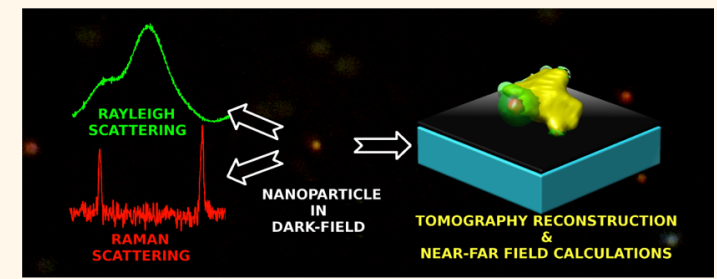
the points of contact with the substrate are of crucial importance for a correct prediction of the NP optical properties. The achieved accuracy of the calculations allows determining how many of the adsorbed molecules have a major contribution to the Raman signal, a fact that has important implications for analyzing experiments and designing sensing applications.
\end{abstract}

KEYWORDS: nanoparticle $\cdot$ enhancement $\cdot$ tomography $\cdot$ DDA $\cdot$ dark-field $\cdot$ Rayleigh $\cdot$ Raman

$\mathrm{C}$ urrently, there is significant scientific and technological interest in the optical properties of noble metal nanoparticles (NPs). In particular, they play a crucial role in the development and optimal design of ultrasensitive spectroscopies including surface-enhanced Raman spectroscopy (SERS), ${ }^{1-10}$ single-molecule SERS, ${ }^{11-16}$ tip-enhanced Raman spectroscopy, ${ }^{17-23}$ surface-enhanced fluorescence, ${ }^{24-27}$ as well as in the detection of chemical and biological agents, ${ }^{28-32}$ in nanolithography, ${ }^{33-36}$ and in the rational design of tips for scanning near-field optical microscopy ${ }^{37-39}$ and fieldenhanced scanning optical microscopy. ${ }^{40}$

The remarkable optical properties of noble metal NPs are dominated by the excitation of localized surface plasmon resonances (LSPRs). LSPRs originate from collective oscillations of the conduction band electrons in a NP and are extremely sensitive to the exact shape, size, composition of the NP, and the surrounding medium.
Complex-shaped NPs are particularly attractive for applications since their LSPRs are widely tunable and they exhibit large field enhancements. ${ }^{41,42}$ Ensembles of complexshaped NPs, however, usually exhibit large dispersity in size and shape. For a detailed understanding of the optical properties of complex-shaped NPs, it is therefore crucial to correlate optical and morphological properties on the single-particle level. ${ }^{43}$

Despite significant progress in experimental single NP studies, most calculations rely on an idealized picture of the real NP, which is obtained either by a 2D morphological characterization or an incomplete 3D characterization from 2D transmission electron microscopy (TEM), scanning tunneling microscopy, ${ }^{44}$ or scanning force microscopy ${ }^{45}$ images.

Electron tomography is well-established as a high-resolution 3D morphological reconstruction technique for nanostructures. ${ }^{46}$ Recently, it was combined with the discrete

\begin{abstract}
* Address correspondence to fjaeckel@liv.ac.uk, coronado@fcq.unc.edu.ar, feldmann@Imu.de.
\end{abstract}

Received for review December 6, 2013 and accepted April 30, 2014.

Published online April 30, 2014 10.1021/nn406270z

() 2014 American Chemical Society 
dipole approximation (DDA) algorithm in order to calculate the optical response of a highly irregular NP in a rigorous way. ${ }^{47}$ Significant differences in the nearand far-field optical response of the same NP were found between calculations using an idealized NP morphology and calculations using all details of the 3D morphology. However, no comparison with experiments was performed in that case. A comparison between experimental Rayleigh scattering spectra for unpolarized light and calculations from electron tomography reconstruction with very good agreement was recently performed for core-shell metal NPs. ${ }^{48}$

Here, we combine state of the art experimental techniques for both 3D morphological reconstruction and near- and far-field optical properties measurements of the same NP, with accurate numerical DDA calculations in order to study different LSPRs in a complex-shaped NP. The far- and near-field optical properties are experimentally quantified by Rayleigh and Raman scattering spectra of the same individual NP for different incident polarizations. The NP morphology is reconstructed, and its points of contact with the substrate are determined by means of $3 D$ electron tomography. The reconstructed NP morphology and its exact position on the substrate serve as input for the DDA calculations. We find that for a correct calculation of the NP optical properties both its exact 3D morphology and its surroundings including the contact points with the substrate are essential. Furthermore, we quantitatively calculate Raman enhancement factors and the number of molecules that contribute to the Raman signal in excellent agreement with the experimental estimates.

To the best of our knowledge, this is the first time that a quantitative characterization of the nearand far-field optical properties of LSPRs of an individual complex-shaped NP is performed by means of Rayleigh and Raman scattering spectroscopy, electron tomography reconstruction, and accurate numerical DDA calculations. As a consequence of the study, the importance of the substrate for the LSPR of a NP is directly demonstrated and the number of molecules with significant contributions to the Raman signal is determined.

\section{RESULTS AND DISCUSSION}

A flow-chart diagram of our combined experimental and theoretical approach is displayed in Figure 1. Complex-shaped gold NPs are functionalized with 4-mercaptobenzoic acid (MBA) on their surface ${ }^{41}$ and deposited on a TEM grid in order to perform consecutive optical spectroscopy and electron tomography on the same individual NP. Polarization-resolved Raman and white light Rayleigh scattering of individual functionalized NPs are measured (step 1 in Figure 1). After the optical measurements, a 3D morphological characterization of the same NP is performed (step 2 in

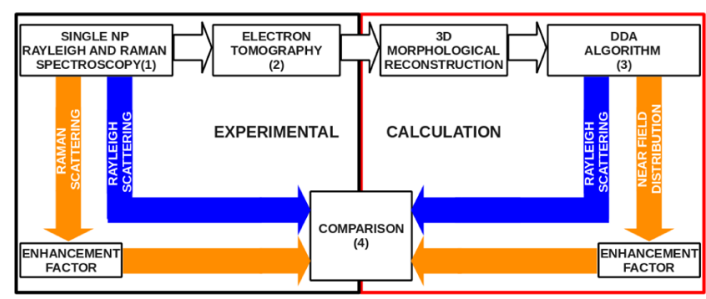

Figure 1. Flow-chart diagram of the combined experimental and theoretical approach.

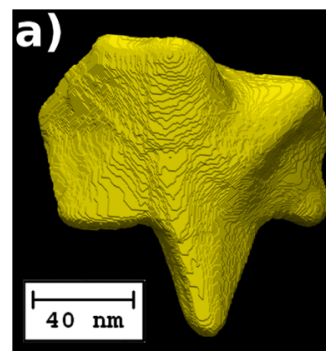

c)
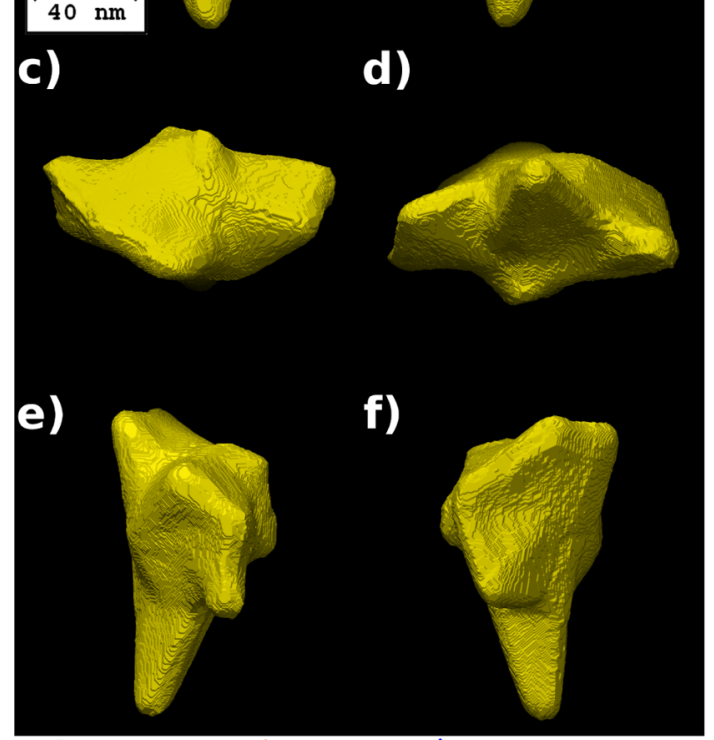

g)

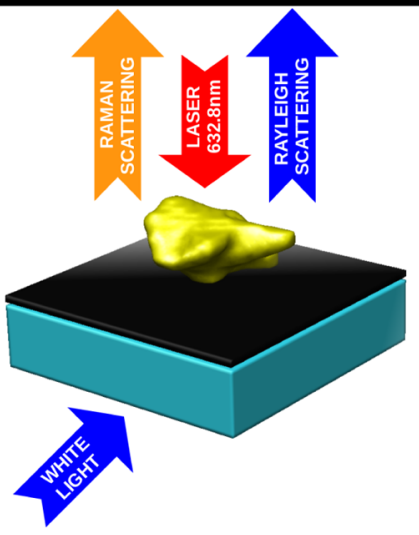

Figure 2. (a-f) Morphological reconstruction of the studied NP which is obtained from a 3D electron tomography. (a) Top view. (b) Bottom view. The red circles indicate the contact points of the NP with the substrate. $(c-d),(e-f)$ Opposite lateral views of the NP. (g) Studied NP on the simulated substrate of which the dimensions are $250 \mathrm{~nm}$ in edge length and $70 \mathrm{~nm}$ total thickness $(10 \mathrm{~nm}$ of carbon (black) which is in contact with the NP and $60 \mathrm{~nm}$ of Formvar (blue)). The direction of the white light, laser, and the collection direction as used in the experiment is also indicated. 

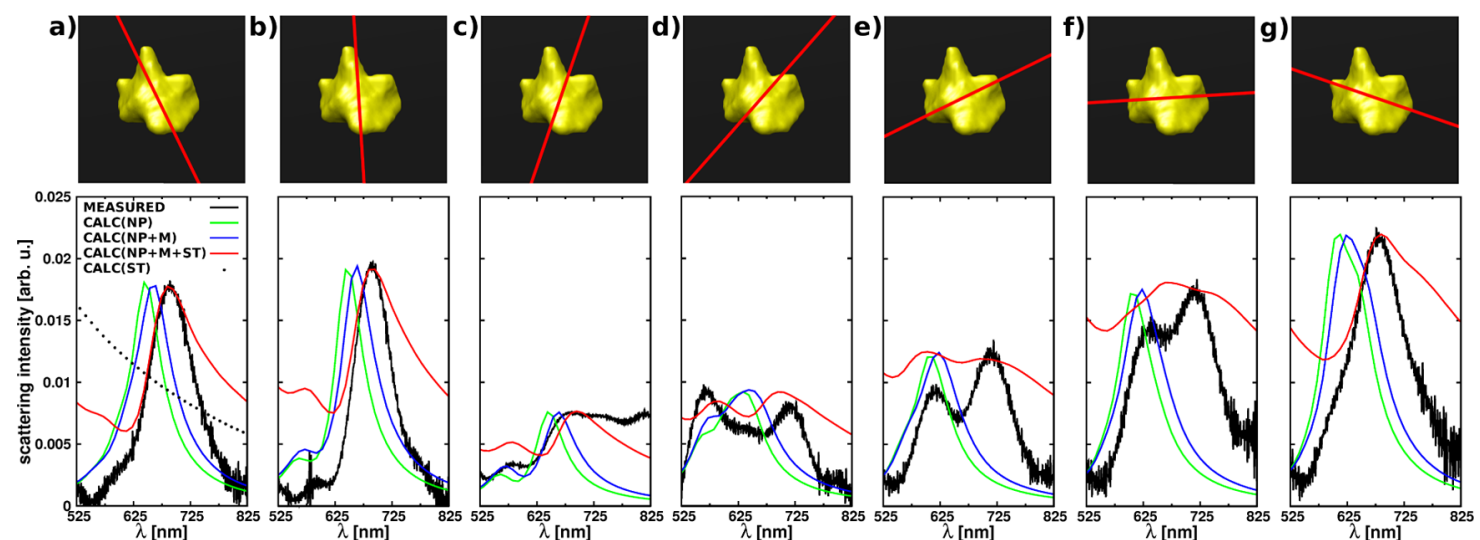

Figure 3. $(\mathrm{a}-\mathrm{g})$ Measured and simulated Rayleigh scattering spectra for each incident polarization which is indicated with a red line in the corresponding image in the top panel of each figure. Black curves: measured scattering spectra (integration time $=120 \mathrm{~s}$ ). Green curves: simulated scattering spectra without any substrate and molecules. Blue curves: simulated scattering spectra considering the MBA molecules. Red curves: simulated scattering spectra considering the MBA molecules and the substrate of Figure $2 \mathrm{~g}$.

Figure 1). The reconstructed 3D morphology is used as input ${ }^{47}$ for DDA calculations (step 3 in Figure 1). In our DDA algorithm, the NP is described as a cubic array of $N$ polarizable elements such that its $3 \mathrm{D}$ morphology is exactly reproduced. ${ }^{47}$

In the following, we first present the exact reconstructed morphology of the studied NP. Subsequently, we compare the measured and calculated Rayleigh scattering spectra of the NP and discuss the near-field optical properties, which are obtained from Raman scattering spectra (step 4 Figure 1).

The exact shape of the studied NP which is obtained from the 3D morphological reconstruction is shown in Figure 2. Panels $a$ and $b$ depict the top and bottom view of the NP, respectively, while panels $c, d$ and e,f show opposite lateral views of the NP. Clearly, the NP has a highly complex and anisotropic shape that cannot be inferred from conventional TEM or scanning probes techniques. The contact points of the NP with the substrate (membrane of the TEM grid) have been identified in the tomography reconstruction and are indicated with red circles in Figure $2 \mathrm{~b}$. Figure $2 \mathrm{~g}$ displays the exact position adopted by the NP on the TEM grid membrane.

We now turn to the discussion of the far-field properties of the NP, that is, the measured and calculated Rayleigh scattering (Figure 3). It is important to note that incident unpolarized light has been excluded in the Rayleigh scattering measurements by introducing a polarizer and an entrance slit with relative perpendicular orientation before the dark-field condenser. Also, a complex-shaped NP gives rise to large changes of its scattering spectrum for small changes in the incident polarization direction. This is very important since many different resonances can be excited by changing the incident polarization.

Black curves in Figure 3 show the measured scattering spectra at different white light incident polarizations. From left to right, the polarization varies in steps of $\pi / 8 \mathrm{rad}$ clockwise, and it is indicated with a red line on the top view of the reconstructed NP above each measured spectrum. Calculated spectra from DDA taking into account the full morphological details of the reconstructed NP (see Methods section) are shown in green (without any substrate and adsorbed molecules), blue (with adsorbed molecules), and red (with adsorbed molecules and substrate).

The comparison of the measured and calculated spectra provides clear evidence for the importance of including both the precise morphology of the NP and the surroundings in the electrodynamic calculations. The peaks in the scattering spectra correspond to the LSPRs of the NP. In general, the spectral position of the resonances can only be reproduced well if the substrate and MBA layer are included. Similarly, the splitting of the measured spectrum into two peaks (Figure $3 d-f$ ) is only reproduced by including the substrate.

In addition to the spectral position of the LSPRs, another important aspect is their spectral widths. In Figure $3 a, b, g$, the measured scattering spectra are dominated by a strong resonance, of which the spectral width is smallest for the polarization direction parallel to the sharpest tip of the NP (Figure 3b). The narrowing of this resonance is well-reproduced for the theoretical calculations as the calculated spectra in the figures show.

On the other hand, our results show that the maximum scattering intensity of the LSPRs increases/ decreases as the mean NP length along the polarization direction increases/decreases. This can be clearly seen in the sequence of spectra in Figure $3 c-g$, for instance. This sequence, however, also displays some differences between calculated and experimental results. Particularly, for the polarizations in Figure $3 d-f$, the relative intensities of the two resonances are opposite in experiment and theory, and in some cases, there are differences between calculated and 


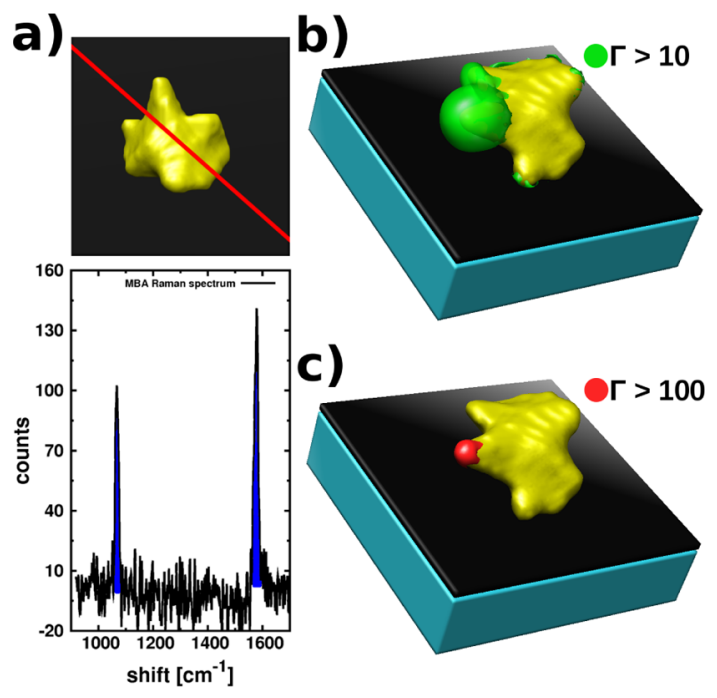

Figure 4. (a) Measured Raman spectrum at laser power = $50 \mu \mathrm{W}$ and wavelength $=632.8 \mathrm{~nm}$. The incident polarization is indicated with a red line in the image above the spectrum. The integrated area under the peaks used for the enhancement factor calculation is indicated in blue. $(b, c) \Gamma$ distribution around the NP at the laser wavelength for values of $\Gamma>10$ and $\Gamma>100$, respectively.

measured peak positions. These differences can be attributed to the differences in incident polarization used in experiment and theory, respectively. In the calculations, a perfectly well-defined incident linear polarization is considered. In the experiment, however, the dark-field configuration will always produce a range of polarizations at the sample even if a polarizer and entrance slit before the condenser is used.

It should be noted that calculated spectra including the substrate show increased intensities at both long and short wavelength tails. This effect is attributed to the finite size of the substrate used in the calculations. The finite substrate scatters light (finite substrate effect) as illustrated by the substrate spectrum (black dots) in Figure 3a. Apparently, the short and long wavelength tails of the calculated spectra including the substrate (red curves) follow the mathematical form of the substrate scattering (black dots) in Figure 3a. Therefore, in order to analyze the scattering spectra with the substrate of Figure 3 , it is necessary to consider that the tails of these spectra should decay as the calculated scattering spectra without the substrate. The finite substrate effect is also responsible of the differences between the relative intensities of the two peaks in calculated and experimental spectra of Figure $3 d-f$.

The optical near-field properties were experimentally characterized by detecting the Raman signals of the adsorbed MBA molecules on the NP surface. Figures $4 \mathrm{a}$ and $5 \mathrm{a}$ show the measured Raman spectra for the incident polarizations, which are indicated with a red line in the top images of each figure. By comparing both measured Raman spectra, it can be noticed

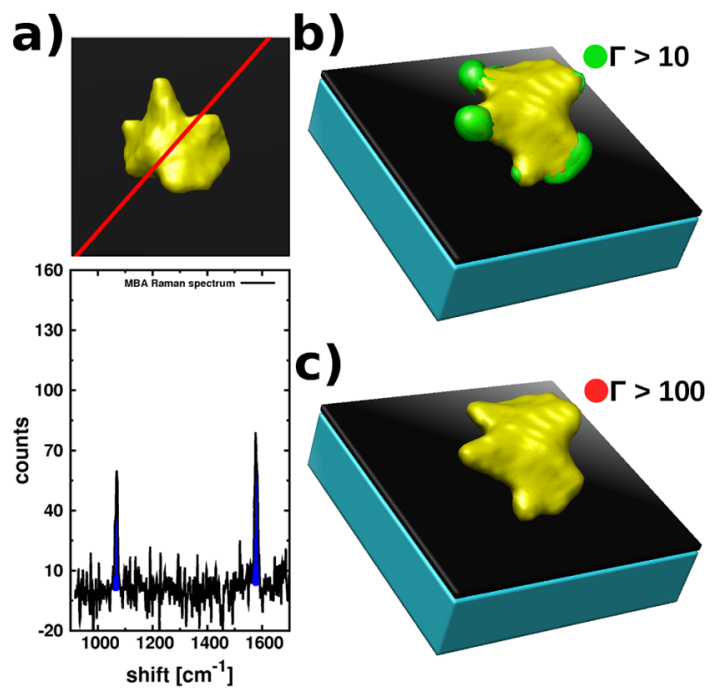

Figure 5. (a) Measured Raman spectrum at laser power = $50 \mu \mathrm{W}$ and wavelength $=632.8 \mathrm{~nm}$. The incident polarization is indicated with a red line in the image above the spectrum. The integrated area under the peaks used for the enhancement factor calculation is indicated in blue. (b,c) $\Gamma$ distribution around the NP at the laser wavelength for values of $\Gamma>10$ and $\Gamma>100$, respectively. Note that there are no values of $\Gamma>100$ around the NP.

that the Raman intensity of the molecules around the NP for the incident polarization of Figure $4 a$ is larger than that for Figure 5a. We emphasize here that great care was taken to reduce any possible photochemical or photothermal degradation of the MBA molecules. Specifically, only three laser shots of $60 \mathrm{~s}$ are used. Two Raman spectra at perpendicular laser polarization are collected from the first and second shot. The third shot, with polarization equal to the first one, is used to confirm that no photochemical degradation occurred; that is no significant differences in the Raman spectrum are measured. A low laser power is necessary to avoid any damage of the TEM grid membrane, the molecules around the NP, and the NP itself (LSPR heating induces melting and reshaping). This low laser power also ensures that no Raman signals from molecules which are not located in high field enhancement regions are detected.

In order to explain the observed excitation polarization dependence, calculations of the near-field properties were performed with DDA and probe dipoles. ${ }^{49}$ As in the far-field case, all experimental details are included in the calculations (see Methods section). Figures $4 \mathrm{~b}$ and $5 \mathrm{~b}$ show the calculated near-field enhancement $(\Gamma)$ distributions around the NP at the laser wavelength for values of $\Gamma>10\left(\Gamma=\left(\mathbf{E} \cdot \mathbf{E}^{*}\right) /\left(\mathbf{E}_{0} \cdot \mathbf{E}_{0}^{*}\right){ }^{49}\right.$ where $\mathbf{E}$ is the local complex electromagnetic field, $\mathbf{E}_{0}$ is the incident field, and $*$ denotes complex conjugated value). In Figure $4 b$, the $\Gamma$ distribution is highly concentrated on the sharpest tip of the NP, whereas in Figure $5 b$, the distribution is more spread out over several tips of the NP. This stronger confinement of the electric field gives rise to much higher values 
of the electromagnetic field enhancement. These features can be clearly seen by comparing the $\Gamma$ distributions at the laser wavelength for $\Gamma>100$ (Figures 4c and $5 \mathrm{c}$ ). High enhancement regions are only found for the former polarization. ${ }^{50}$ This fact is in qualitative agreement with the higher intensity of the Raman spectrum in Figure 4a compared to Figure 5a.

In order to obtain a quantitative comparison between near-field calculations and Raman spectra, the SERS substrate enhancement factor $(\mathrm{Y})^{51}$ is calculated. It should be noted that the term substrate in $\mathrm{Y}$ corresponds to NP surface as the MBA molecules are adsorbed to the NP. As the molecular orientation is unknown and may fluctuate during a measurement, the expression for $\mathrm{Y}$ (see eq 4.10 of ref 51 ) is

$$
\mathrm{Y}=\frac{1}{A_{\mathrm{M}}} \int \Theta(r) \mathrm{d} S=\{\Theta\}
$$

where $\Theta$ is the orientation-averaged single-molecule enhancement factor which depends on the position $r$ of the molecule on the NP surface $\left(A_{M}\right)$.

Note that $\mathrm{Y}$ is the mean value of $\Theta$ on the NP surface and is linked to the Raman signals by (see eq 4.13 of ref 51)

$$
I_{\mathrm{SERS}}=\mu_{\mathrm{S}} A_{\mathrm{M}}\{\Theta\} \frac{\mathrm{d} \sigma_{\mathrm{RS}}}{\mathrm{d} \Omega} S_{\text {Inc }} \Delta \Omega_{\text {Det }}
$$

where $\mu_{\mathrm{S}}$ is the surface density of molecules, $S_{\text {Inc. }}$ the incident Poynting vector, $\mathrm{d} \sigma_{\mathrm{RS}} / \mathrm{d} \omega$ the differential Raman cross section of the molecule, and $\Delta \Omega_{\text {Det }}$ is the solid angle of the collection cone, which is given by

$$
\Delta \Omega_{\text {Det }}=2 \pi\left(1-\cos \left(\arcsin \left(\frac{\mathrm{NA}}{n_{\mathrm{M}}}\right)\right)\right)
$$

where NA is the numerical aperture of the objective and $n_{\mathrm{M}}$ is the refractive index of the medium. Furthermore, the detection efficiency $(\eta)$ of the experimental setup needs to be taken into account

$$
\{\Theta\}=\frac{I_{\text {SERS }}}{\eta \mu_{\mathrm{S}} A_{\mathrm{M}} \frac{\mathrm{d} \sigma_{\mathrm{RS}}}{\mathrm{d} \Omega} S_{\mathrm{Inc}} \Delta \Omega_{\text {Det }}}
$$

At any given time, each MBA molecule will have a particular position and orientation on the NP surface. However, the detector collects Raman signals from many molecules with different orientations and positions. As the total number of molecules is large, we assume that the same mean value $\{\Theta\}$ can be obtained by considering many molecules around the NP with an isotropic Raman tensor and a single-molecule enhancement factor (SMEF) equal to $\operatorname{SMEF}(r)=\Theta(r)$. Going beyond the $|E|^{4}$ approximation by approximating the radiation enhancement $\left(\Gamma_{\text {Rad }}\right)$ by the local field enhancement $\Gamma_{\text {Rad }}(\lambda) \approx \Gamma(\lambda)$ for the $\operatorname{SMEF}$ (i.e., SMEF $\approx$ $\Gamma\left(\lambda_{1}\right) \Gamma\left(\lambda_{p}\right)$, where $\lambda_{1}$ is the laser wavelength and $\lambda_{p}$ is the Raman signal wavelength), the $\{\Theta\}$ can be rewritten as

$$
\{\Theta\} \approx \frac{1}{N} \sum_{i=1}^{N} \Gamma_{i}\left(\lambda_{1}\right) \Gamma_{i}\left(\lambda_{\mathrm{p}}\right)
$$

where $N$ is the number of dipoles of the emulated MBA layer in DDA calculations, $\Gamma_{i}\left(\lambda_{1}\right)$ is the enhancement factor for dipole $i$ at the laser wavelength and $\Gamma_{i}\left(\lambda_{p}\right)$ is the enhancement factor for dipole $i$ at the wavelength of the peak $\mathrm{p}$ of the Raman spectrum.

In eq $4, I_{\text {SERS }}$ is calculated by integrating the most reliable peaks ( 1070 and $1577 \mathrm{~cm}^{-1}$ that correspond to $\lambda_{1}=679 \mathrm{~nm}$ and $\lambda_{2}=703 \mathrm{~nm}$, respectively) of the measured Raman spectra and by considering the acquisition time $t=60 \mathrm{~s}$. The integrated areas are indicated in Figures $4 \mathrm{a}$ and $5 \mathrm{a}$. For our experimental setup, $\eta=0.56$ and $\Delta \Omega_{\text {Det }}=2.13$, and a value of $S_{\text {Inc }}=$ $2500 \mathrm{w} / \mathrm{cm}^{2}$ is calculated according to Gaussian beam optics. A value of $A_{M}=27740 \mathrm{~nm}^{2}$ is calculated from the morphological reconstruction and by taking into account a packing density of $0.19 \mathrm{~nm}^{2}$ per molecule ${ }^{41}$ or $\mu_{\mathrm{S}}=5.263 \mathrm{~nm}^{-2}$, the number of molecules on the NP is $\mu_{S} A_{M}=146000$. The Raman cross sections for the MBA molecule are determined from $a b$ initio calculations $s^{52,51}$ (see Theoretical Calculations section) and correspond to $\mathrm{d} \sigma_{\mathrm{RS}} / \mathrm{d} \Omega=1.945 \times 10^{-30} \mathrm{~cm}^{2}$ and $\mathrm{d} \sigma_{\mathrm{RS}} / \mathrm{d} \Omega=4.480 \times 10^{-30} \mathrm{~cm}^{2}$ for the Raman modes 1070 and $1577 \mathrm{~cm}^{-1}$, respectively. For the polarization in Figure 4, we find $I_{\text {SERS }}=1.05 \times 10^{-17} \mathrm{~W}$ and the measured $\{\Theta\}=12410$ for the Raman mode $1070 \mathrm{~cm}^{-1}$ and $I_{\text {SERS }}=1.23 \times 10^{-17} \mathrm{~W}$ and $\{\Theta\}=6293$ for the Raman mode $1577 \mathrm{~cm}^{-1}$. On the other hand, evaluating eq 5 by using the results of the near-field calculations at the laser wavelength in addition to calculated $\Gamma$ distributions to the Raman peak wavelengths $\lambda_{1}=679 \mathrm{~nm}$ and $\lambda_{2}=703 \mathrm{~nm}$, we find calculated values $\{\Theta\}=11312$ and 7957 for the Raman modes 1070 and $1577 \mathrm{~cm}^{-1}$, respectively.

Applying the same analysis to the polarization in Figure 5 , we find $I_{\text {SERS }}=3.46 \times 10^{-18} \mathrm{~W}$ and the measured $\{\Theta\}=4081$ and $I_{\text {SERS }}=5.43 \times 10^{-18} \mathrm{~W}$ and $\{\Theta\}=$ 2780 for the Raman modes 1070 and $1577 \mathrm{~cm}^{-1}$, respectively. The calculated values from eq 5 are $\{\Theta\}=1630$ and 1456 for the Raman modes 1070 and $1577 \mathrm{~cm}^{-1}$, respectively. The agreement of measured and calculated $\{\Theta\}$ values is excellent, validating the DDA calculations combined with 3D morphological reconstruction as a powerful tool to predict near-field optical properties of complexshaped metal NPs.

We now take the analysis a step further by determining how many molecules are producing most of the Raman signals. To this end, we calculate an effective area $\left(A_{\text {eff }}\right)^{53}$ based on the $\Gamma$ distribution around the NP. This area represents the region on the NP surface where most of the field enhancement is concentrated 
and consequently most of the Raman signal is produced. The effective area is calculated according to

$$
A_{\text {eff }}=d^{2} \sum_{i=1}^{N} \frac{\Gamma_{i}^{2}}{\Gamma_{\max }^{2}}
$$

where $N$ is the number of dipoles emulating the MBA layer, $\Gamma_{i}$ is the enhancement factor for each dipole, and $d$ is the grid size parameter of the cubic array. For the polarization in Figure 4, we find $A_{\text {eff }}=14.3 \mathrm{~nm}^{2}$, which corresponds to only 75 molecules generating most of Raman response. Equation 6 implies that these molecules are located in regions of the highest enhancement. In this case, this region is the sharpest tip of the NP (compare Figure 4c).

Introducing $A_{\text {eff }}$ instead of $A_{M}$ in eq 4, values of $\{\Theta\}=$ $1.37 \times 10^{7}$ and $6.94 \times 10^{6}$ are obtained for the Raman modes 1070 and $1577 \mathrm{~cm}^{-1}$, respectively. Applying the same procedure to the polarization in Figure 5, we find $A_{\text {eff }}=25.17 \mathrm{~nm}^{2}$, and therefore, about 132 molecules are generating most of the Raman signal with $\{\Theta\}=4.50 \times 10^{6}$ for the Raman mode $1070 \mathrm{~cm}^{-1}$ and $\{\Theta\}=3.06 \times 10^{6}$ for Raman mode $1577 \mathrm{~cm}^{-1}$. The order of magnitude for these enhancement factors is in good agreement with earlier estimates of local enhancement factors of complex-shaped gold nanostars. ${ }^{41}$

\section{CONCLUSIONS}

In conclusion, we have performed a rigorous experimental and theoretical study of the far- and near-field optical properties of a complex-shaped NP. White light Rayleigh and Raman scattering spectra were measured at the single-NP level, and 3D electron tomography was performed to reconstruct the real NP morphology and identify the NP points of contact with the substrate. The morphological data were used as input for DDA calculations.

The comparison between simulated and measured Rayleigh spectra at different incident polarizations demonstrates the excellent potential of DDA combined with precise knowledge of morphology obtained by electron tomography, for the simulation of the optical properties of complex-shaped metal NPs. The comparison further shows the importance of including the exact shape, as well as the surroundings (substrate and MBA layer) in the calculations. This leads

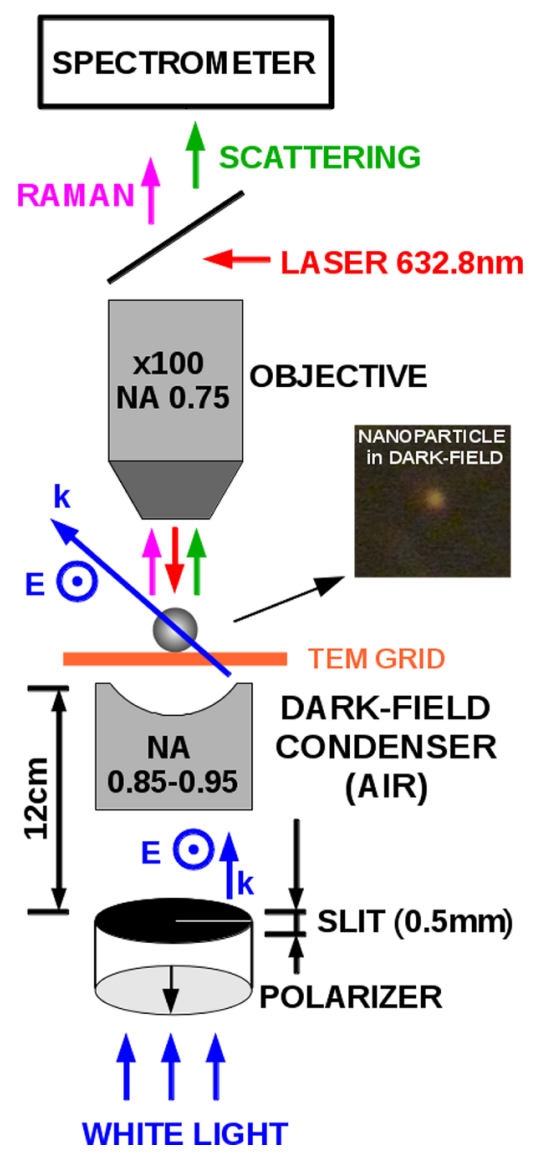

Figure 6. Setup of the combined dark-field and Raman scattering microscope which is used to perform the optical property measurements. An image of the studied NP in the dark-field is also shown.

to strongly improved predictions of far-field optical properties for a real NP.

Near-field distributions around the NP were assessed from Raman spectra of MBA molecules on the NP surface measured for two polarizations by determining enhancement factors for each excitation. The experimentally determined enhancement factors are accurately reproduced by the theoretical results, demonstrating the high performance of DDA calculations combined with electron tomography to predict nearfield optical properties, as well. Finally, the good agreement between theory and experiments allowed determining the number of molecules on the NP producing most of the Raman signals.

\section{METHODS}

Optical Characterization. The complex-shaped gold NPs were synthesized via the seeded growth method. ${ }^{54}$ The NPs are functionalized with 4-mercaptobenzoic acid (MBA) on their surface $^{41}$ and deposited on a marked TEM grid (Tedpella 01802-F). Polarization-resolved Raman and white light Rayleigh scattering of individual functionalized NPs were measured on a combined dark-field and Raman scattering microscope (Figure 6). ${ }^{41}$ Well-defined polarized excitation at the sample in dark-field configuration was achieved by placing a polarizer and a narrow entrance slit before the dark-field condenser. Before analysis, Raman spectra were corrected for background and high-frequency noise in both Rayleigh and Raman spectra was reduced by using a fast Fourier transform low-pass filter.

Morphological Reconstruction. After the optical measurements, a 3D morphological characterization of the same NP was performed by using high-angle annular dark-field scanning transmission electron microscopy. Images were recorded at tilt 
increments of $2^{\circ}$ between $\pm 70^{\circ}$. Alignment was accomplished via ImageJ, ${ }^{55}$ using the TomoJ add-on ${ }^{56}$ which comprises a landmark-based alignment script. ${ }^{57}$ The actual morphological reconstruction was performed by a masked simultaneous iterative reconstruction technique step to obtain an adequate segmentation and a good starting model, followed by a discrete algebraic reconstruction technique step. ${ }^{58}$

Theoretical Calculations. The reconstructed 3D morphology was used as input for DDA calculations. In our DDA algorithm, the NP was described as a cubic array of $N$ polarizable elements such that its 3D morphology was exactly reproduced. ${ }^{47}$ The response of the dipole array to an applied electromagnetic field was calculated by determining the induced dipole moment in each element self-consistently. The near-field around the NP was calculated by using probe dipoles. ${ }^{49}$ The substrate was modeled as a film having $250 \mathrm{~nm}$ edge length and a total thickness of $70 \mathrm{~nm}$ (10 nm of carbon which is in direct contact with the NP and $60 \mathrm{~nm}$ of Formvar). These thicknesses correspond to TEM grid specifications of the manufacturer, and the edge length $(250 \mathrm{~nm})$ was limited by the computational effort. The MBA molecules adsorbed on the NP surface were emulated by $\mathrm{a} \approx 1 \mathrm{~nm}$ thick layer of DDA dipoles. The polarizabilities of the dipoles in the cubic array were based on tabulated dielectric constants for gold ${ }^{59}$ and carbon. ${ }^{60}$ For Formvar and MBA, refractive indexes of $1.475^{61}$ and $1.45^{62}$ were used, respectively. Approximately $1.5 \times 10^{6}$ and $6 \times 10^{6}$ dipoles were used for scattering and field enhancement calculations, respectively. The calculations considered the incident wave vector direction of the experiment and perfect linear polarizations. The Raman cross sections of the MBA molecule were calculated with Gaussian 09, revision $B .01^{52}$ by B3LYP/6-311++g(d,p). Among the calculated Raman modes close to the experimental ones of 1070 and $1577 \mathrm{~cm}^{-1}$, the calculated modes of 1111 and $1634 \mathrm{~cm}^{-1}$ were used to determine the Raman cross sections (see eq A.10 of ref 51) as they present the highest Raman activity.

Conflict of Interest: The authors declare no competing financial interest.

Acknowledgment. The authors wish to acknowledge the assistance of the Sistema Nacional de Microscopía (SNM), and Consejo Nacional de Investigaciones Científicas y Técnicas (CONICET), and Secretaría de Ciencia y Tecnología de la Universidad Nacional de Córdoba (SECYT-UNC) and FONCYT Agencia Nacional de Promoción Científica y Tecnológica, as well as the Deutsche Forschungsgemeinschaft (DFG) through the Nanosystems Initiative Munich (NIM), and the European Research Council (ERC) through the Advanced Investigator Grant HYMEM for financial support. The authors would like to thank C. Capello for technical assistance with the $a b$ initio Gaussian calculations.

\section{REFERENCES AND NOTES}

1. Ou, F. S.; Hu, M.; Naumov, I.; Kim, A.; Wu, W.; Bratkovsky, A. M.; Li, X.; Williams, R. S.; Li, Z. Hot-Spot Engineering in Polygonal Nanofinger Assemblies for Surface Enhanced Raman Spectroscopy. Nano Lett. 2011, 11, 2538-2542.

2. Osberg, K. D.; Rycenga, M.; Harris, N.; Schmucker, A. L.; Langille, M. R.; Schatz, G. C.; Mirkin, C. A. Dispersible Gold Nanorod Dimers with Sub-5 nm Gaps as Local Amplifiers for Surface-Enhanced Raman Scattering. Nano Lett. 2012, $12,3828-3832$

3. Rumyantseva, A.; Kostcheev, S.; Adam, P. M.; Gaponenko, S. V.; Vaschenko, S. V.; Kulakovich, O. S.; Ramanenka, A. A.; Guzatov, D. V.; Korbutyak, D.; Dzhagan, V.; et al. Nonresonant Surface-Enhanced Raman Scattering of ZnO Quantum Dots with Au and Ag Nanoparticles. ACS Nano 2013, 7, 3420-3426.

4. D'Andrea, C.; Bochterle, J.; Toma, A.; Huck, C.; Neubrech, F.; Messina, E.; Fazio, B.; Marag, O. M.; Di Fabrizio, E.; Lamy de La Chapelle, M.; et al. Optical Nanoantennas for Multiband Surface-Enhanced Infrared and Raman Spectroscopy. ACS Nano 2013, 7, 3522-3531.

5. Ando, J.; Fujita, K.; Smith, N. I.; Kawata, S. Dynamic SERS Imaging of Cellular Transport Pathways with
Endocytosed Gold Nanoparticles. Nano Lett. 2011, 11, 5344-5348.

6. Shegai, T.; Brian, B.; Miljković, V. D.; Käll, M. Angular Distribution of Surface-Enhanced Raman Scattering from Individual Au Nanoparticle Aggregates. ACS Nano 2011, 5 , 2036-2041.

7. Tong, L.; Righini, M.; Gonzalez, M. U.; Quidant, R.; Käll, M. Optical Aggregation of Metal Nanoparticles in a Microfluidic Channel for Surface-Enhanced Raman Scattering Analysis. Lab Chip 2009, 9, 193-195.

8. Zhang, Q.; Large, N.; Nordlander, P.; Wang, H. Porous Au Nanoparticles with Tunable Plasmon Resonances and Intense Field Enhancements for Single-Particle SERS. J. Phys. Chem. Lett. 2014, 5, 370-374.

9. Li, L.; Steiner, U.; Mahajan, S. Single Nanoparticle SERS Probes of Ion Intercalation in Metal-Oxide Electrodes. Nano Lett. 2014, 14, 495-498.

10. Salehi, M.; Steinigeweg, D.; Ströbel, P.; Marx, A.; Packeisen, J.; Schülcker, S. Rapid Immuno-SERS Microscopy for Tissue Imaging with Single-Nanoparticle Sensitivity. J. Biophotonics 2013, 6, 785-792.

11. Le Ru, E. C.; Etchegoin, P. G. Single-Molecule SurfaceEnhanced Raman Spectroscopy. Annu. Rev. Phys. Chem. 2012, 63, 65-87.

12. Willets, K. A.; Stranahan, S. M.; Weber, M. L. Shedding Light on Surface-Enhanced Raman Scattering Hot Spots through Single-Molecule Super-Resolution Imaging. J. Phys. Chem. Lett. 2012, 3, 1286-1294.

13. Patra, P. P.; Kumar, G. V. P. Single-Molecule SurfaceEnhanced Raman Scattering Sensitivity of Ag-Core AuShell Nanoparticles: Revealed by Bi-analyte Method. J. Phys. Chem. Lett. 2013, 4, 1167-1171.

14. Le Ru, E. C.; Grand, J.; Sow, I.; Somerville, W. R. C.; Etchegoin, P. G.; Treguer Delapierre, M.; Charron, G.; Flidj, N.; Lvi, G.; Aubard, J. A Scheme for Detecting Every Single Target Molecule with Surface-Enhanced Raman Spectroscopy. Nano Lett. 2011, 11, 5013-5019.

15. Lee, J. H.; Nam, J. M.; Jeon, K. S.; Lim, D. K.; Kim, H.; Kwon, S.; Lee, H.; Suh, Y. D. Tuning and Maximizing the SingleMolecule Surface-Enhanced Raman Scattering from DNATethered Nanodumbbells. ACS Nano 2012, 6, 9574-9584.

16. Lee, K.; Irudayaraj, J. Correct Spectral Conversion between Surface-Enhanced Raman and Plasmon Resonance Scattering from Nanoparticle Dimers for Single-Molecule Detection. Small 2013, 9, 1106-1115.

17. Kawata, S. Plasmonics for Nanoimaging and Nanospectroscopy. Appl. Spectrosc. 2013, 67, 117-125.

18. Zhang, R.; Zhang, Y.; Dong, Z.; Jiang, S.; Zhang, C.; Chen, L.; Zhang, L.; Liao, Y.; Aizpurua, J.; Luo, Y.; et al. Chemical Mapping of a Single Molecule by Plasmon-Enhanced Raman Scattering. Nature 2013, 498, 82-86.

19. Sonntag, M. D.; Klingsporn, J. M.; Garibay, L. K.; Roberts, J. M.; Dieringer, J. A.; Seideman, T.; Scheidt, K. A.; Jensen, L.; Schatz, G. C.; Van Duyne, R. P. Single-Molecule TipEnhanced Raman Spectroscopy. J. Phys. Chem. C 2012, $116,478-483$

20. Jiang, N.; Foley, E. T.; Klingsporn, J. M.; Sonntag, M. D.; Valley, N. A.; Dieringer, J. A.; Seideman, T.; Schatz, G. C.; Hersam, M. C.; Van Duyne, R. P. Observation of Multiple Vibrational Modes in Ultrahigh Vacuum Tip-Enhanced Raman Spectroscopy Combined with Molecular-Resolution Scanning Tunneling Microscopy. Nano Lett. 2012, 12, 50615067.

21. Wood, B. R.; Asghari Khiavi, M.; Bailo, E.; McNaughton, D.; Deckert, V. Detection of Nano-oxidation Sites on the Surface of Hemoglobin Crystals Using Tip-Enhanced Raman Scattering. Nano Lett. 2012, 12, 1555-1560.

22. Paulite, M.; Blum, C.; Schmid, T.; Opilik, L.; Eyer, K.; Walker, G. C.; Zenobi, R. Full Spectroscopic Tip-Enhanced Raman Imaging of Single Nanotapes Formed from $\beta$-Amyloid(1-40) Peptide Fragments. ACS Nano 2013, 7, 911-920.

23. Pozzi, E. A.; Sonntag, M. D.; Jiang, N.; Klingsporn, J. M.; Hersam, M. C.; Van Duyne, R. P. Tip-Enhanced Raman Imaging: An Emergent Tool for Probing Biology at the Nanoscale. ACS Nano 2013, 7, 885-888. 
24. Kim, K.; Lee, J. W.; Shin, K. S. Polyethylenimine-Capped Ag Nanoparticle Film as a Platform for Detecting Charged Dye Molecules by Surface-Enhanced Raman Scattering and Metal-Enhanced Fluorescence. ACS Appl. Mater. Interfaces 2012, 4, 5498-5504.

25. Zhang, Z.; Yang, P.; Xu, H.; Zheng, H. Surface Enhanced Fluorescence and Raman Scattering by Gold Nanoparticle Dimers and Trimers. J. Appl. Phys. 2013, 113, 033102.

26. Le Ru, E. C.; Etchegoin, P. G.; Grand, J.; Flidj, N.; Aubard, J.; Lvi, G. Mechanisms of Spectral Profile Modification in Surface-Enhanced Fluorescence. J. Phys. Chem. C 2007, 111, 16076-16079.

27. Gabudean, A. M.; Focsan, M.; Astilean, S. Gold Nanorods Performing as Dual-Modal Nanoprobes via Metal-Enhanced Fluorescence (MEF) and Surface-Enhanced Raman Scattering (SERS). J. Phys. Chem. C 2012, 116, 12240-12249.

28. Yang, X.; Gu, C.; Qian, F.; Li, Y.; Zhang, J. Z. Highly Sensitive Detection of Proteins and Bacteria in Aqueous Solution Using Surface-Enhanced Raman Scattering and Optical Fibers. Anal. Chem. 2011, 83, 5888-5894.

29. Haes, A. J.; Chang, L.; Klein, W. L.; Van Duyne, R. P. Detection of a Biomarker for Alzheimer's Disease from Synthetic and Clinical Samples Using a Nanoscale Optical Biosensor. J. Am. Chem. Soc. 2005, 127, 2264-2271.

30. Sherry, L. J.; Jin, R.; Mirkin, C. A.; Schatz, G. C.; Van Duyne, R. P. Localized Surface Plasmon Resonance Spectroscopy of Single Silver Triangular Nanoprisms. Nano Lett. 2006, 6, 2060-2065.

31. Tittl, A.; Yin, X.; Giessen, H.; Tian, X. D.; Tian, Z. Q.; Kremers, C.; Chigrin, D. N.; Liu, N. Plasmonic Smart Dust for Probing Local Chemical Reactions. Nano Lett. 2013, 13, 1816-1821.

32. Anker, J. N.; Hall, W. P.; Lyandres, O.; Shah, N. C.; Zhao, J.; Van Duyne, R. P. Biosensing with Plasmonic Nanosensors. Nat. Mater. 2008, 7, 442-453.

33. Srituravanich, W.; Fang, N.; Sun, C.; Luo, Q.; Zhang, X. Plasmonic Nanolithography. Nano Lett. 2004, 4, 1085-1088.

34. Sundaramurthy, A.; Schuck, P. J.; Conley, N. R.; Fromm, D. P.; Kino, G. S.; Moerner, W. E. Toward Nanometer-Scale Optical Photolithography: Utilizing the Near-Field of Bowtie Optical Nanoantennas. Nano Lett. 2006, 6, 355-360.

35. Kim, S.; Jung, H.; Kim, Y.; Jang, J.; Hahn, J. W. Resolution Limit in Plasmonic Lithography for Practical Applications beyond 2x-nm Half Pitch. Adv. Mater. 2012, 24, OP337OP344.

36. Ozbay, E. Plasmonics: Merging Photonics and Electronics at Nanoscale Dimensions. Science 2006, 311, 189-193.

37. Jandard, F.; Fauquet, C.; Dehlinger, M.; Ranguis, A.; Bjeoumikhov, A.; Ferrero, S.; Pailharey, D.; Dahmani, B.; Tonneau, D. Mapping of X-ray Induced Luminescence Using a \{SNOM\} Probe. Appl. Surf. Sci. 2013, 267, 81-85.

38. Novotny, L.; Stranick, S. J. Near-Field Optical Microscopy and Spectroscopy with Pointed Probes. Annu. Rev. Phys. Chem. 2006, 57, 303-331.

39. Frey, H. G.; Witt, S.; Felderer, K.; Guckenberger, R. HighResolution Imaging of Single Fluorescent Molecules with the Optical Near-Field of a Metal Tip. Phys. Rev. Lett. 2004, 93, 200801.

40. Perassi, E. M.; Scarpettini, A. F.; Masip, M. E.; Bragas, A. V.; Coronado, E. A. Understanding the Behavior of New Plasmonic Probes with Sub-nanometric Resolution in Field Enhanced Scanning Optical Microscopy. J. Phys. Chem. C 2011, 115, 10455-10461.

41. Hrelescu, C.; Sau, T. K.; Rogach, A. L.; Jäckel, F.; Feldmann, J. Single Gold Nanostars Enhance Raman Scattering. Appl. Phys. Lett. 2009, 94, 153113.

42. Sau, T. K.; Rogach, A. L.; Jäckel, F.; Klar, T. A.; Feldmann, J. Properties and Applications of Colloidal Nonspherical Noble Metal Nanoparticles. Adv. Mater. 2010, 22, 18051825.

43. Henry, A. I.; Bingham, J. M.; Ringe, E.; Marks, L. D.; Schatz, G. C.; Van Duyne, R. P. Correlated Structure and Optical Property Studies of Plasmonic Nanoparticles. J. Phys. Chem. C 2011, 115, 9291-9305.

44. Kern, A. M.; Meixner, A. J.; Martin, O. J. F. MoleculeDependent Plasmonic Enhancement of Fluorescence and Raman Scattering near Realistic Nanostructures. ACS Nano 2012, 6, 9828-9836.

45. Shao, L.; Susha, A. S.; Cheung, L. S.; Sau, T. K.; Rogach, A. L.; Wang, J. Plasmonic Properties of Single Multispiked Gold Nanostars: Correlating Modeling with Experiments. Langmuir 2012, 28, 8979-8984.

46. Saghi, Z.; Midgley, P. A. Electron Tomography in the (S)TEM: From Nanoscale Morphological Analysis to 3D Atomic Imaging. Annu. Rev. Mater. Res. 2012, 42, 59-79.

47. Perassi, E. M.; Hernandez Garrido, J. C.; Moreno, M. S.; Encina, E. R.; Coronado, E. A.; Midgley, P. A. Using Highly Accurate 3D Nanometrology To Model the Optical Properties of Highly Irregular Nanoparticles: A Powerful Tool for Rational Design of Plasmonic Devices. Nano Lett. 2010, 10, 2097-2104.

48. Chuntonov, L.; Bar-Sadan, M.; Houben, L.; Haran, G. Correlating Electron Tomography and Plasmon Spectroscopy of Single Noble Metal CoreShell Nanoparticles. Nano Lett. 2012, 12, 145-150.

49. Perassi, E. M.; Canali, L. R.; Coronado, E. A. Enhancement and Confinement Analysis of the Electromagnetic Fields Inside Hot Spots. J. Phys. Chem. C 2009, 113, 6315-6319.

50. Hrelescu, C.; Sau, T. K.; Rogach, A. L.; Jäckel, F.; Laurent, G.; Douillard, L.; Charra, F. Selective Excitation of Individual Plasmonic Hotspots at the Tips of Single Gold Nanostars. Nano Lett. 2011, 11, 402-407.

51. Le Ru, E.; Etchegoin, P. Principles of Surface Raman Spectroscopy; Elsevier: Oxford, 2009.

52. Frisch, M. J.; Trucks, G. W.; Schlegel, H. B.; Scuseria, G. E.; Robb, M. A.; Cheeseman, J. R.; Scalmani, G.; Barone, V.; Mennucci, B.; Petersson, G. A.; et al. Gaussian 09, revision D.01; Gaussian Inc.: Wallingford, CT, 2009.

53. Schuck, P. J.; Fromm, D. P.; Sundaramurthy, A.; Kino, G. S.; Moerner, W. E. Improving the Mismatch between Light and Nanoscale Objects with Gold Bowtie Nanoantennas. Phys. Rev. Lett. 2005, 94, 017402.

54. Sau, T. K.; Murphy, C. J. Room Temperature, High-Yield Synthesis of Multiple Shapes of Gold Nanoparticles in Aqueous Solution. J. Am. Chem. Soc. 2004, 126, 86488649.

55. Rasband, W. ImageJ.; National Institutes of Health: Bethesda, MD, 1997-2011.

56. Messaoudil, C.; Boudier, T.; Sorzano, C.; Marco, S. TomoJ: Tomography Software for Three-Dimensional Reconstruction in Transmission Electron Microscopy. BMC Bioinf. 2007, 8, 288.

57. Sorzano, C.; Messaoudi, C.; Eibauer, M.; Bilbao Castro, J.; Hegerl, R.; Nickell, S.; Marco, S.; Carazo, J. Marker-Free Image Registration of Electron Tomography Tilt-Series. BMC Bioinf. 2009, 10, 124.

58. Zürner, A.; Döblinger, M.; Cauda, V.; Wei, R.; Bein, T. Discrete Tomography of Demanding Samples Based on a Modified SIRT Algorithm. Ultramicroscopy 2012, 115, 41-49.

59. Palik, E. D. Handbook of Optical Constant of Solids; Academic Press: New York, 1985.

60. Arakawa, E. T.; Dolfini, S. M.; Ashley, J. C.; Williams, M. W. Arc-Evaporated Carbon Films: Optical Properties and Electron Mean Free Paths. Phys. Rev. B 1985, 31, 8097-8101.

61. Shukla, R.; Chowdhury, A.; Gupta, P. D. Interferometric Determination of Thickness of Free-Standing Submicron Formvar Films. Opt. Eng. 1994, 33, 1881-1884.

62. Barriet, D.; Yam, C. M.; Shmakova, O. E.; Jamison, A. C.; Lee, T. R. 4-Mercaptophenylboronic Acid SAMs on Gold: Comparison with SAMs Derived from Thiophenol, 4-Mercaptophenol, and 4-Mercaptobenzoic Acid. Langmuir 2007, 23, 8866-8875. 